\title{
Mapping and candidate gene screening of tomato Cladosporium fulvum-resistant gene Cf-19, based on high-throughput sequencing technology
}

Tingting Zhao, Jingbin Jiang, Guan Liu, Shanshan He, He Zhang, Xiuling Chen, Jingfu Li and Xiangyang Xu*

\begin{abstract}
Background: Tomato leaf mold is a common disease in tomato cultivation. This disease is caused by Cladosporium fulvum, which has many physiological races and differentiates rapidly. Cf genes confer resistance to C. fulvum, and the C. fulvum-tomato pathosystem is a model for the study of gene-for-gene interactions. Plants carrying the Cf-19 gene show effective resistance to $C$. fulvum in the field, and can be used in breeding and resistance mechanism studies as new resistant materials. In this study, we used $F_{2}$ bulk specific-locus amplified fragment sequencing (SLAF-seq) and parental resequencing methods to locate and characterize the Cf-19 gene.
\end{abstract}

Results: A total of 4108 Diff_markers and three association regions were found in association analysis. A 2.14-Mb region containing seven Cf-type genes was identified in further analysis based on data from SLAF-seq and parental resequencing. Two candidate genes, Solyc01g006550.2.1 and Solyc01g005870.1.1, were screened out by quantitative real-time PCR (qRT-PCR) analysis. Sequence analysis showed that Solyc01 g006550.2.1 (an allelic locus of Cf-0) in CGN18423 was a novel homologue of the Cladosporium resistance gene Cf-9 (Hcr9s) in the Cf-4/9 locus. The marker P7, which cosegregated with the resistant trait, was developed based on sequence mutation of the Solyc01g006550.2.1 locus in CGN18423.

Conclusions: The Cf-19 gene was mapped to the short arm of chromosome 1. The candidate genes Solyc01g006550.2.1 and Solyc01g005870.1.1 showed related amino acid sequence structures and expression patterns. Solyc01g006550.2.1 had a close evolutionary relationship with the functional Hcr9 members Cf-4 and Cf-9, and was very different from non-functional members. The results from this study will facilitate the breeding of cultivars carrying the Cf-19 gene and provide a basis for further gene cloning, resistance gene evolution and plant resistance mechanism studies.

Keywords: Cf-19 gene, Cf-4/9 locus, Solanum lycopersicum, Cladosporium fulvum, Tomato leaf mold, SLAF-seq

\section{Background}

Tomato leaf mold disease, caused by the biotrophic fungus Cladosporium fulvum, is a serious disease of Solanum lycopersicum (tomato) [1]. This disease can reduce both fruit yield and quality, and sometimes even kill tomato plants. In compatible interactions with susceptible tomato plants, fungal spores germinate on the abaxial surface of leaves and enter the leaf apoplast through stomata. Hyphae emerge through the stomata

\footnotetext{
* Correspondence: xxy709@126.com

College of Horticulture, Northeast Agricultural University, Harbin 150030,
} China cells undergo necrosis [2]. Tomato $C f$ genes confer resistance to $C$. fulvum and mediate incompatible interactions between $C$. fulvum and tomato plants. In incompatible interactions, fungal hyphae are arrested in their development soon after penetration of the sub-stomatal cavity, and their growth is restricted to limited necrotic lesions [2]. Plant breeders introduced $C f$ resistance genes from wild Solanum species into cultivated tomato to control the disease many years ago [3, 4], and this is still an efficient method in tomato cultivation today. A number of $C f$ genes have 
been introgressed for use in commercial breeding, but this artificial selection has created evolutionary pressure on $C$. fulvum. To date, many $C f$ genes have been overcome by C. fulvum carrying matching avirulence genes (Avr). A race that has evolved to overcome the resistance genes $C f$ 2, $C f-4, C f-5, C f-9$, and $C f-11$ was reported by Lindhout et al. [5]. In northeast China, the C. fulvum physiological races 1.2.3.4 and 1.2.4 have evolved to overcome the $C f-4$ gene and become the dominant races in only nine years [6]. The number of $C f$ genes that can be used in breeding is decreasing, making it necessary to identify new resistance genes and breed them into tomato cultivars.

$C f$ genes, which encode predicted membrane-bound proteins with extracytoplasmic leucine-rich repeats (LRRs) [7], confer resistance to specific races of C. fulvum through recognition of $A v r$ peptides secreted into the leaf apoplast during infection [8]. In the tomato-C. fulvum interaction, a strict correlation exists between the triggering of a hypersensitive response (HR) and resistance, as the various Avrs induce a specific HR in tomato genotypes carrying a matching $C f$ resistance gene [9]. In 1980, $24 C f$ genes located on 12 chromosomes were reported in tomato $[10,11]$. Later studies showed that the $C f$ genes were organized into clusters of resistance gene homologues, which have been designated Hcr2s and Hcr9s for homologues of the Cladosporium resistance genes $C f-2$ and $C f-9$, respectively [12]. $H c r 2$ loci, including the $C f-2$ and $C f-5$ loci, have been mapped to the short arm of chromosome 6 [13]. Three Hcr2 homologues were found at the $C f-2$ locus. The $C f-2.1$ and $C f-2.2$ genes are nearly identical, and are functional genes that confer resistance to strains of C. fulvum that carry the matching Avr2 gene. The other homologue $(H c r 2-2 A)$ is not functional [14]. At the $C f-5$ locus, four homologues were found, among which $\mathrm{Hcr} 2-5 \mathrm{C}$ is the corresponding functional gene $C f-5$ [15]. The $C f-4$ and $C f-9$ loci, each comprising five Hcr9s, have been mapped to the short arm of chromosome 1 [16]. Hcr $9-4 D$ and $H c r 9-9 C$ are the functional genes $C f-4$ and $C f-9$, respectively $[7,17]$. Tomato plants carrying the $C f-19$ gene have shown efficient resistance in the field and no infection has been reported for this gene to date. $C f-19$ was assigned to the long arm of chromosome 2 in 1980 [11]. Our previously study showed that $C f-19$ was a dominant gene [18] and induced a remarkable HR in tomato plants inoculated with $C$. fulvum physiological race 1.2.3.4, indicating that it is a functional member of the $C f$ gene family.

SLAF-seq, which is based on high-throughput sequencing, is a recently developed high-resolution strategy for large-scale de novo single nucleotide polymorphism (SNP) discovery and genotyping [19]. This method is relatively low-cost and efficient, and can be used for gene and quantitative trait locus (QTL) mapping. The efficiency of this approach was tested in rice and soybean, and it was successfully used to construct genetic maps for sesame (Sesamum indicum L.) [20]. We applied this approach to an $\mathrm{F}_{2}$ population in combination with genome resequencing of the parents to map and characterize the $C f-19$ gene rapidly.

\section{Results}

Disease severity ratings and genetic analysis of the Cf-19 gene

CGN18423 and $F_{1}$ plants were resistant to the C. fulvum race 1.2.3.4, while Moneymaker plants were susceptible. Chi-square analysis showed that the segregation ratio of the resistant and susceptible individuals of the $\mathrm{F}_{2}$ population was $3: 1$. Resistant and susceptible $\mathrm{BC}_{1}$ plants segregated according to the expected ratio of 1:1 (Table 1).

\section{High-throughput sequencing analysis}

A total of $20.15 \mathrm{~Gb}$ data including $99.75 \mathrm{M}$ reads and $4.07 \mathrm{~Gb}$ data including $20.22 \mathrm{M}$ reads were obtained from parental genome sequencing and $F_{2}$ bulk SLAFseq, respectively (Table 2). From the 119,504 SLAF tags, 4108 Diff-markers were obtained. A distribution diagram of the markers on each chromosome was drawn according to the results of SLAF positioning on the genome (Fig. 1).

\section{Association analysis and candidate gene screening}

According to the results of $\triangle \mathrm{SNP}$ index calculation, all Diff-Markers were distributed on chromosome 1. Regions with three or more consecutive Diff-markers were regarded as association regions. We found three association regions including 203 Diff-markers and 244 genes on chromosome 1 in SLAF-seq analysis (Table 3). To further narrow the mapping region, SNPs in the association regions from parental resequencing were analyzed in combination with the $F_{2}$ bulk SLAF-seq data. Forty-three SNPs showed the Moneymaker base type in the susceptible pool, and both parental base types in the resistant pool were screened out. Of the 43 SNPs, 34 were distributed in an approximately $2.14-\mathrm{Mb}$ region in association region I, one was in association region II and eight were in association region III (Additional file 1: Table S1). Further analysis was carried out based on the results of association

Table 1 Genetic analysis of Cf-19 disease resistance in different generations

\begin{tabular}{|c|c|c|c|c|c|}
\hline \multirow[t]{2}{*}{ Generation } & \multirow[b]{2}{*}{ Total } & \multicolumn{2}{|l|}{ No. of plants } & \multirow{2}{*}{$\begin{array}{l}\text { Expected } \\
\text { segregation } \\
\text { ratio (R:S) }\end{array}$} & \multirow[t]{2}{*}{$x^{2}$} \\
\hline & & Resistant (R) & Susceptible (S) & & \\
\hline CGN18423 & 30 & 30 & 0 & & \\
\hline Moneymaker & 30 & 0 & 30 & & \\
\hline $\mathrm{F}_{1}$ & 30 & 30 & 0 & & \\
\hline $\mathrm{F}_{2}$ & 469 & 339 & 130 & $3: 1$ & 1.70 \\
\hline $\mathrm{BC}_{1}$ & 102 & 45 & 57 & $1: 1$ & 1.19 \\
\hline
\end{tabular}

$\mathrm{X}^{2} 0.05,1=3.84$ 
Table 2 Sequencing data of each sample

\begin{tabular}{llllll}
\hline Sample & Sample-ID & Read length & Total reads & Total nucleotides & GC (\%) percentage \\
\hline Resistant pool & aa & $101+101$ & $11,033,299$ & $2,222,039,840$ & $35.39 \%$ \\
Susceptible pool & ab & $101+101$ & $9,184,513$ & $1,851,224,404$ & $35.36 \%$ \\
CGN18423 & M & $101+101$ & $49,250,898$ & $9,948,681,396$ & $36.96 \%$ \\
Moneymaker & P & $101+101$ & $50,497,727$ & $10,200,540,854$ & $37.41 \%$ \\
\hline
\end{tabular}

analysis and gene function annotation. Finally, seven genes with $C f$-type characters were screened out, all of which were inside the 2.14-Mb association region I that was identified in the previous SNP analysis. These genes were Solyc01g005730.2.1, Solyc01g005760.2.1, Solyc 01g005780.1.1, Solyc01g005870.1.1, Solyc01g006550.2.1, Solyc01g008390.1.1 and Solyc01g008410.1.1, respectively (Fig. 2).

\section{Quantitative real-time PCR analysis}

According to the results of qRT-PCR analysis, of the seven candidate genes, two (Solyc01g005870.1.1 and Solyc01g006550.2.1) showed expression patterns related to the resistance response process. As shown in Fig. 3, the Solyc01g006550.2.1 gene was expressed at a low level before inoculation, and increased slightly after inoculation. This expression level was maintained for about 5 days, and then increased rapidly at 7 days after inoculation (DAI) and kept increasing during the following days. The highest expression was at 21 DAI, which was 62 -fold higher than the 0 DAI value. The expression level of the Solyc01g005870.1.1 gene was relatively lower at every stage compared with the corresponding level of Solyc01g006550.2.1, while the general expression patterns of the two genes were similar. All other five genes showed unrelated expression patterns during the whole penetration process.

\section{Candidate loci sequencing and sequence analysis}

The Solyc01g005870.1.1 and Solyc01g006550.2.1 loci of CGN18423 and Moneymaker were sequenced successfully (GenBank: KT874515, KT874514, KT845954, KT874513). Sequence alignment showed that the DNA sequences of both loci contained mutations between CGN18423 and Moneymaker. At the Solyc01g005870.1.1 locus, the encoded protein contained a signal peptide, 19 LRRs and a transmembrane region. Several SNPs existed in the coding region, but no difference was found in the conserved domain distribution between CGN18423 and Moneymaker. At the Solyc01g006550.2.1 locus, a 60-bp insertion was found near the $\mathrm{N}$-terminus of the coding region in CGN18423. This insertion changed the ORF, provided a signal peptide for the original Cf- 0 , and led to an increased number of LRR domains (from 27 to 30), according to conserved domain and feature analysis (Fig. 4).

Multiple DNA sequence alignment of the candidate genes and other $C f-4 / 9$ locus genes showed that the Solyc01g006550.2.1 locus of CGN18423 had a close evolutionary relationship with the $C f-4$ and $C f-9$ genes. The Solyc01g005870.1.1 locus of CGN18423 showed a short evolutionary distance to the $C f-O$ gene, and the level of sequence divergence was very low (Fig. 5).

\section{Marker development and linkage analysis}

A sequence characterized amplified region (SCAR) marker (forward primer: 5'-AGTGCAGAAATGGGTTGT

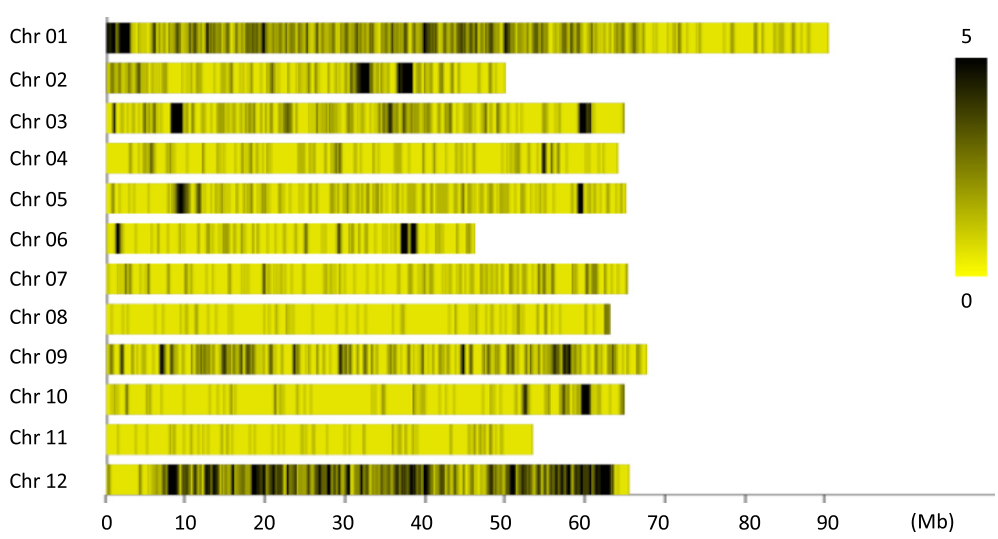

Fig. 1 Marker distribution on the chromosomes. Abscissa: position of SLAF tags on the chromosomes; ordinate: chromosome ID. The darker the color, the more SLAF tags were present 
Table 3 Detail information of association regions

\begin{tabular}{lllcll}
\hline Chromosome ID & Start (genome positon) & End (genome positon) & Size (Mb) & Diff_Marker number & Gene number \\
\hline @SL2.40ch01 & 10,814 & $6,124,581$ & 6.11 & 118 & 236 \\
@SL2.40ch01 & $19,985,817$ & $22,639,492$ & 2.65 & 23 & 2 \\
@SL2.40ch01 & $44,283,583$ & $49,095,823$ & 4.81 & 62 & 6 \\
\hline
\end{tabular}

GTA-3'; reverse primer: 5'-CCGGAGATCAAGCTCAAC CA-3') was found to co-segregate with the resistance trait. This marker was developed based on the 60-bp insertion in the Solyc01g006550.2.1 locus in CGN18423. As shown in Fig. 6, a fragment of 300 bp was amplified in CGN18423, a fragment of 240 bp was amplified in Moneymaker, and both of these fragments were amplified in the $\mathrm{F}_{1}$ samples. Among $345 \mathrm{~F}_{2}$ plants, 72 susceptible plants showed the Moneymaker genotype, 270 resistant plants showed the CGN18423 or $\mathrm{F}_{1}$ genotype, and three susceptible plants showed the $F_{1}$ genotype. In the $F_{3}$ lines test, all $131 \mathrm{~F}_{3}-1$ plants showed the CGN18423 genotype, the $\mathrm{F}_{3}-2$ plants included 42 plants with the CGN18423 genotype, 35 plants with the Moneymaker genotype, and 90 plants with the $F_{1}$ genotype, and all 76 plants of $F_{3}-3$ line showed the Moneymaker genotype. The inoculation and molecular marker identification results of all $F_{3}$ line plants showed consensus.

\section{Discussion}

The $C f$-19 gene was mapped to the short arm of chromosome 1

$C f-19$ was assigned to the long arm of chromosome 2 in 1980 [11], while this gene was mapped at the short arm of chromosome 1 in this study. Although some Diff_Markers were found on the long arm of chromosome 2 according to the results of $\mathrm{F}_{2}$ bulk SLAF-seq analysis, they could not form association regions in further association analysis. Most Diff_Markers were found on the short arm of chromosome 1, and these markers showed us three association regions in association analysis. The position information for these regions was very

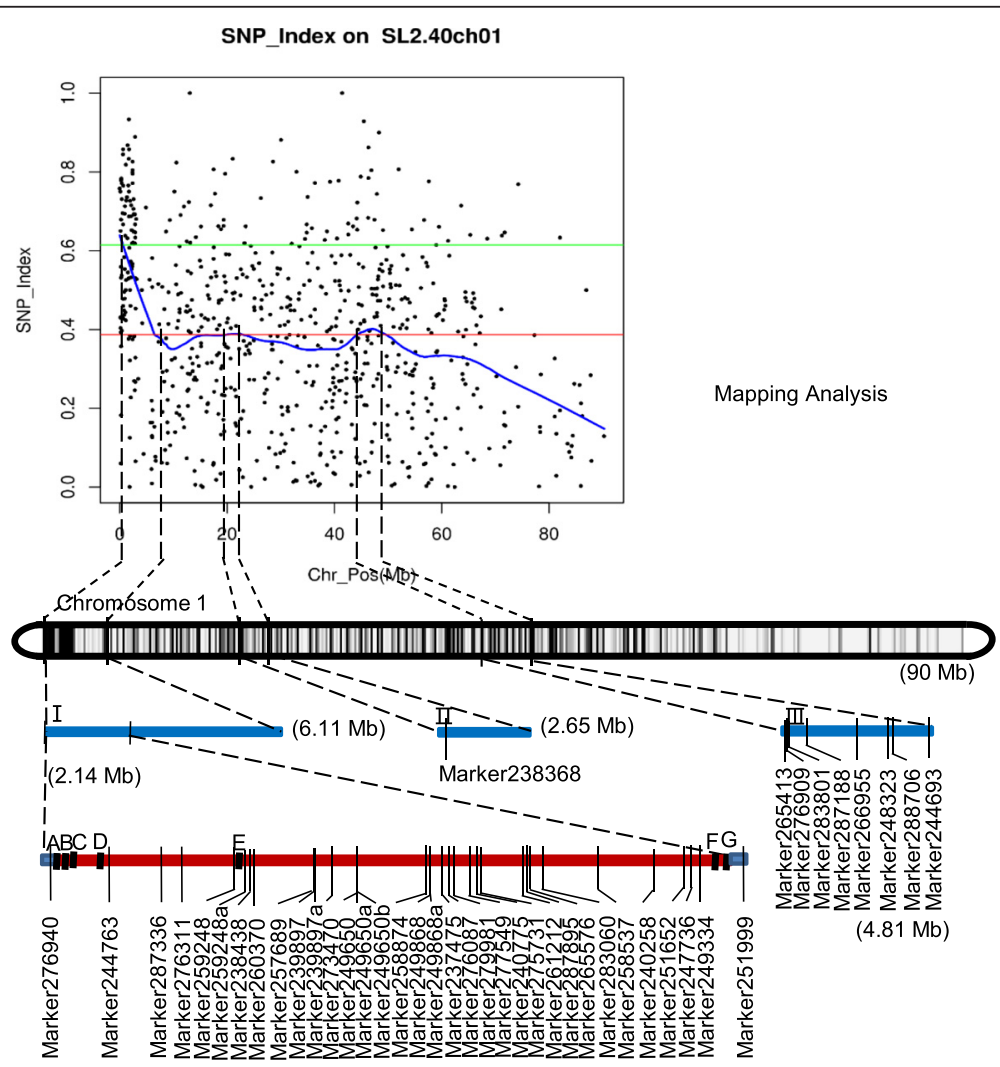

Fig. 2 Genetic and physical maps of mapping regions and the mapping analysis process. Three association regions are shown as I, II and III based on the $F_{2}$ SLAF-seq analysis. These regions are 6.11, 2.65 and $4.81 \mathrm{Mb}$ in size, respectively. Forty-three markers distributed in the three regions and seven candidate genes $(\mathrm{A}-\mathrm{G})$ in a 2.14-Mb region were screened out by the combination of $\mathrm{F}_{2}$ SLAF-seq and parental resequencing. A, Solyc01g005730.2.1; B, Solyc01g005760.2.1; C, Solyc01g005780.1.1; D, Solyc01g005870.1.1; E, Solyc01g006550.2.1; F, Solyc01 g008390.1.1; G, Solyc01g008410.1.1 


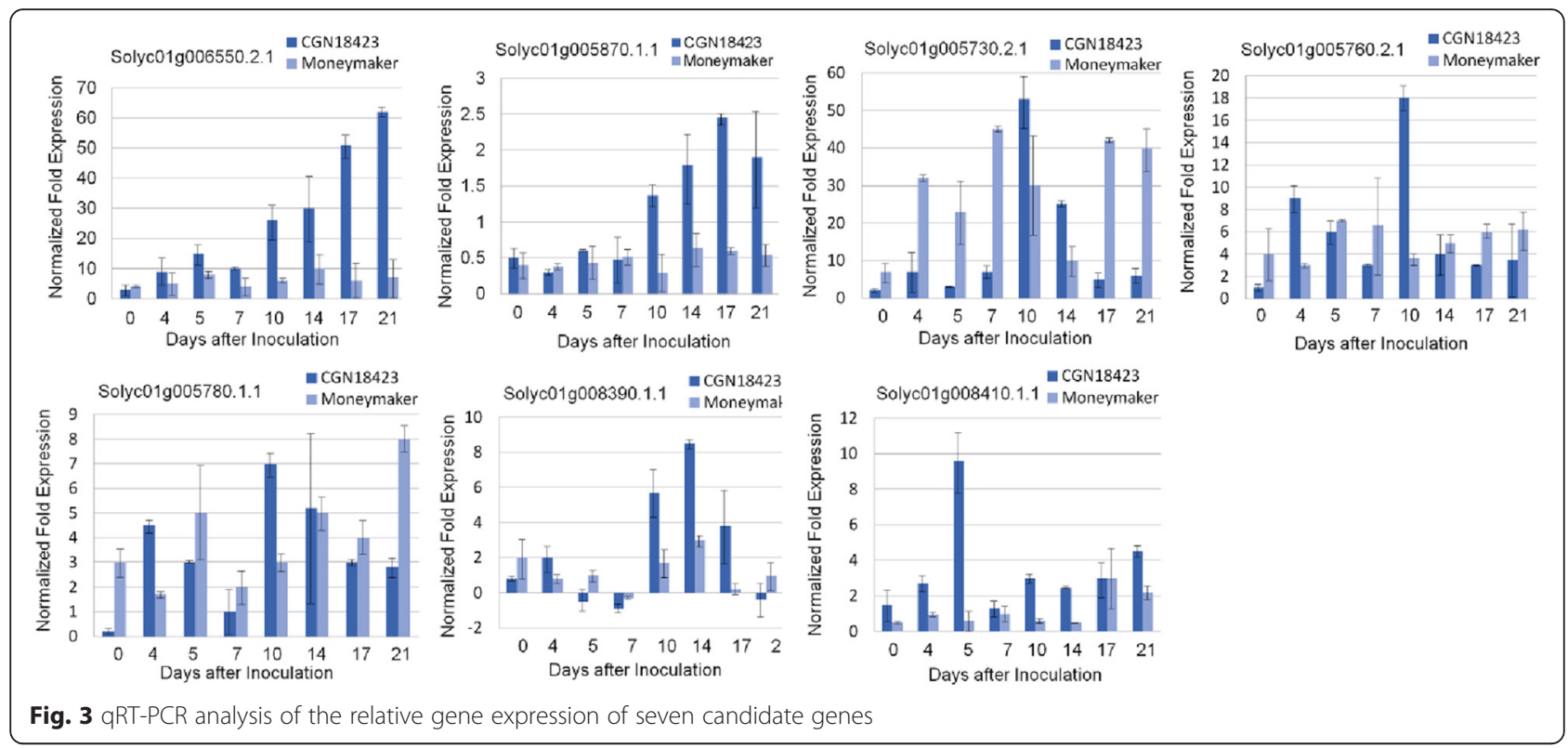

reasonable for the $C f$ genes were organized into clusters of resistance gene homologues and most $C f$ genes mapped to chromosome 1 or chromosome 6 so far.

Three association regions for the $C f-19$ gene were identified in $\mathrm{F}_{2}$ bulk SLAF-seq analysis. This result was not as accurate as we expected. All association regions in this study were on chromosome 1 , and this may have been the reason why the location result was not accurate. The arrangements of $C f$ genes on the short arm of chromosome 1 are very complex. Two loci $(C f-4$ and $C f-9)$ of the clustered $H c r 9$ genes have been mapped to this region, and each comprises five $H c r 9$ s [16]. Additionally, the $C f-1$ gene [21] has been mapped and closely linked to $C f-4 / 9$ [16]. Other studies have mapped the $C f-E C P 1, C f-E C P 2$, $C f$-ECP3, Cf-ECP4 and Cf-ECP5 genes to the short arm of chromosome 1 [22-25]. Many homologous fragments produced by evolutionary events are present in these genes and intergenic regions. These homologous sequences may influence the reads mapping and SNP statistics.

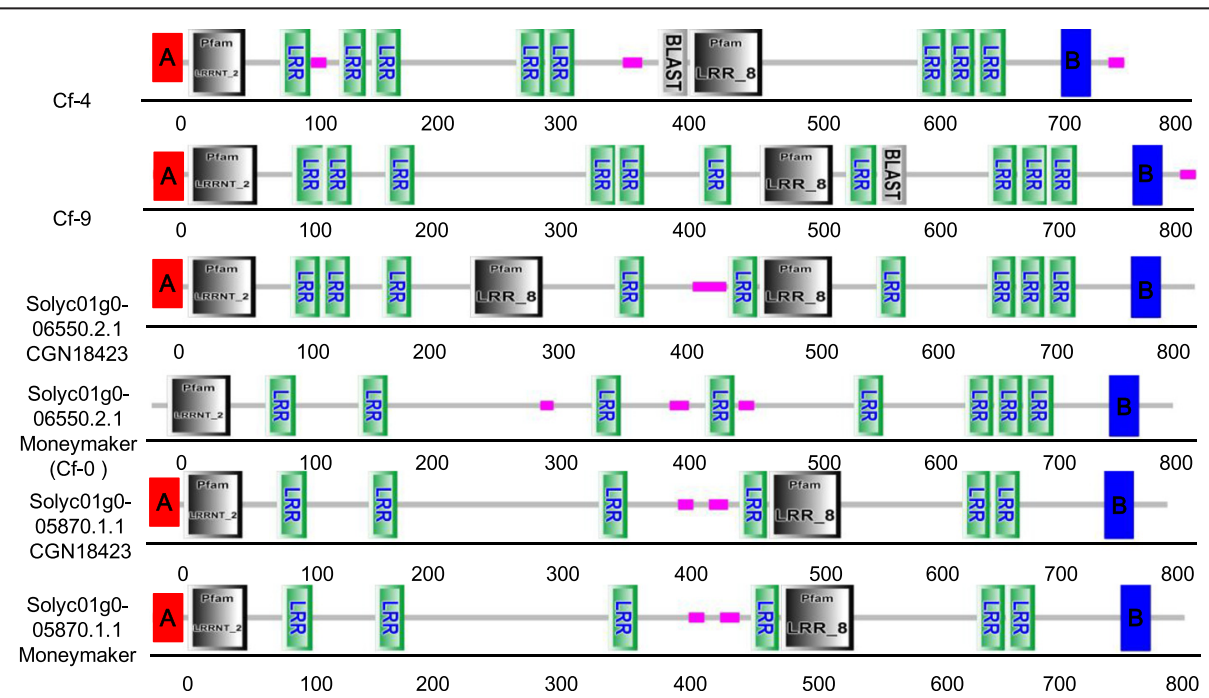

Fig. 4 Protein structure comparative analysis of candidate loci and classical Cf proteins. All proteins contain a signal peptide, leucine-rich repeats (LRR) and a transmembrane region except for Cf-0 of Moneymaker, which lacks the signal peptide domain. The Solyc01g006550.2.1 locus proteins in CGN18423 and Moneymaker are very different in domain type and LRR number, while the Solyc01g005870.1.1 locus proteins in CGN18423 and Moneymaker contain the same domains and LRR number. The red box with the letter " $A$ " inside shows the signal peptide domain and the blue box with the letter "B" inside shows the transmembrane region 


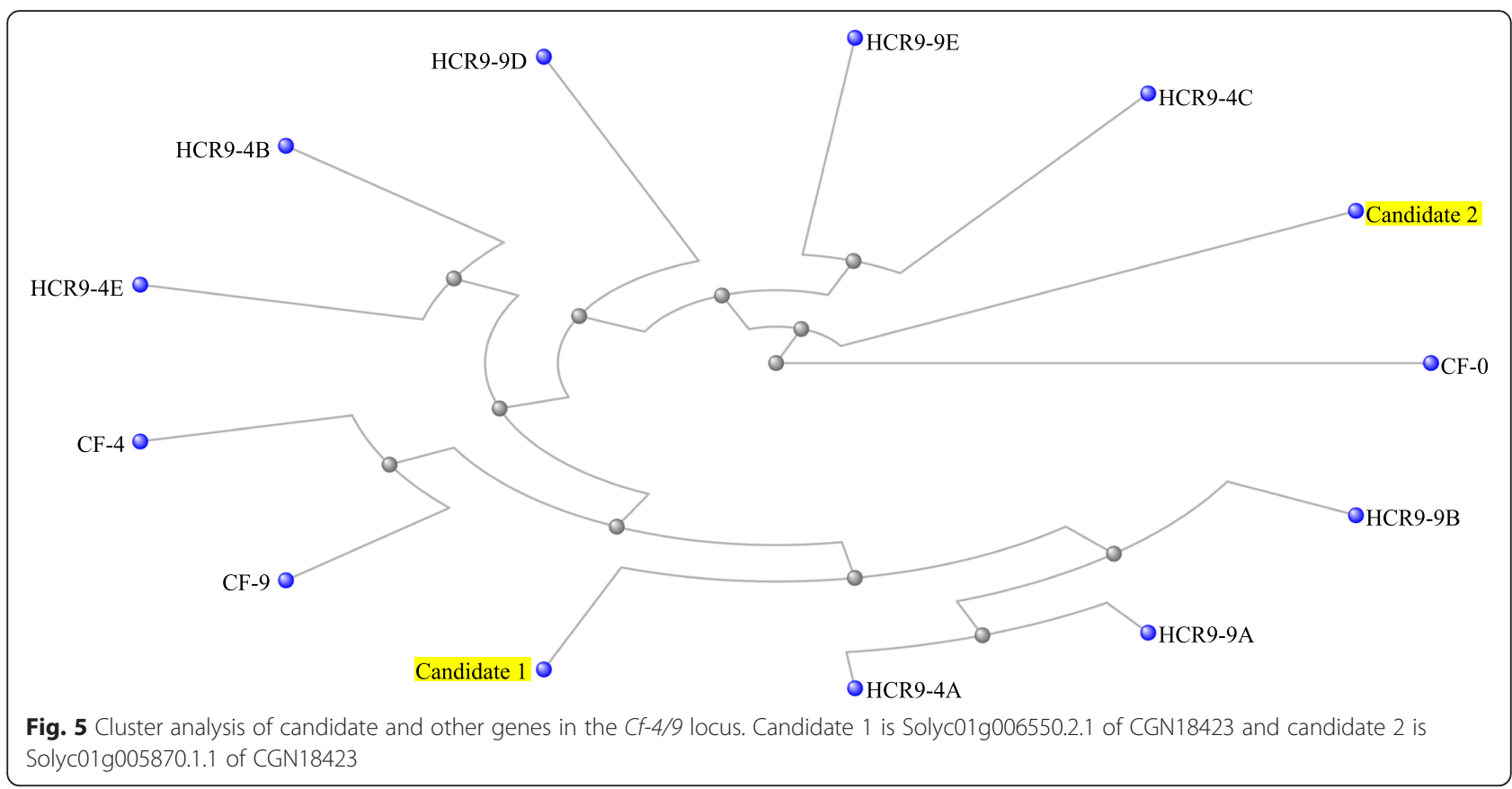

The candidate gene Solyc01g006550.2.1 of CGN18423 is a new $\mathrm{Hcr} 9$ member in the $C f-4 / 9$ locus

The $C f-4$ gene originating from Lycopersicon hirsutum and the $C f-9$ gene originating from $L$. pimpinellifolium were mapped to the $C f-4 / 9$ locus [13]. This locus is flanked by conserved lipoxygenase sequences and is very complex, while only a single $\mathrm{Hcr} 9$ gene has been found at the $C f-4 / 9$ locus in the disease-susceptible cultivar of Lycopersicon esculentum Moneymaker [7]; this gene was designated $C f-0$. The Solyc01g006550.2.1 gene locus in Moneymaker (Solyc01g006550.2.1-Moneymaker) is just the $C f-0$ locus; therefore, our candidate gene Solyc 01g006550.2.1 of CGN18423 (Solyc01g006550.2.1CGN18423) is an allele of $C f-0$. Sequence analysis showed that a 60-bp insertion was present in the $\mathrm{N}$ terminal coding region, and this insertion resulted in changes in the ORF. Blast search results indicated that no genes were completely homologous to the Solyc01g006550.2.1-CGN18423 gene, which suggests that the candidate gene Solyc01g006550.2.1-CGN18423 is a novel member of the $C f-4 / 9$ locus.

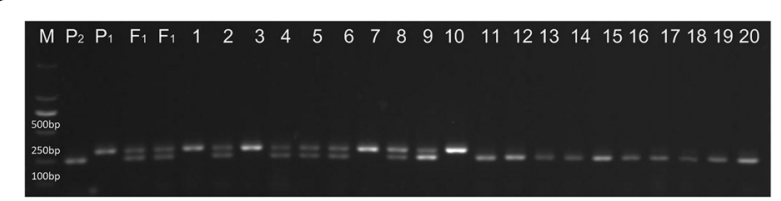

Fig. 6 Marker P7 amplification in different generations. P : CGN18423; $P_{2}$ : Moneymaker; $F_{1}: F_{1}$ plants from the cross of CGN18423 and Moneymaker; 1-10: resistant plants of the $F_{2}$ population; 11-20: susceptible plants of the $F_{2}$ population
$C f$ genes encode proteins with classical signal peptide domains in their N-termini, LRRs, and a transmembrane region in their C-termini. $C f-4$ and $C f-9$ have identical C-termini [14], while a significant degree of sequence divergence is found in their $\mathrm{N}$-terminal portions. This difference between $C f-4$ and $C f-9$ produces their recognition specificity [7]. A similar result was found in this study; the C-terminal portion of the candidate gene Solyc01g006550.2.1-CGN18423 had high identity to Cf-4 and $C f-9$, while the $\mathrm{N}$-terminal portion contained a high degree of sequence divergence. This result indicates that Solyc01g006550.2.1-CGN18423 may have recognition specificity that has formed based on a similar evolutionary mechanism to $C f-4$ and $C f-9$. LRRs can form a $\beta$ strand $/ \beta$-turn motif in which the hypervariable residues are solvent-exposed and potentially contribute to recognition specificity. All $\mathrm{Hcr} 9 \mathrm{~s}$ at the $C f-4 / 9$ locus encode proteins with 27 LRRs with the exception of $4 \mathrm{~B}$, which contains 23 LRRs, and $C f-4$, which comprises 25 LRRs [7]. The candidate gene Solyc01g006550.2.1-CGN18423 encoded 30 LRRs, which is different to all other Hcr9s, providing conditions for the formation of a specific motif for ligand recognition. Phylogenetic analysis showed that the candidate gene Solyc01g006550.2.1-CGN18423 had very short evolutionary distances to functional $\mathrm{Hcr} 9 \mathrm{~s}$ including Cf-4, Cf-9, Hcr 9-9A and Hcr9-9B (adult plants carrying $9 A$ and $9 B$ showed a resistance response to $C$. ful$v u m$ ); these genes were clustered in a branch with a high degree of evolution, and were distinct from other nonfunctional $H c r 9$ s. This result suggested a high possibility that the candidate gene Solyc01g006550.2.1-CGN18423 was our target gene $C f-19$. 


\section{The marker P7 can be used in marker-assisted selection} (MAS) breeding

P7 is a codominant marker that co-segregates with the resistance trait. This marker was designed based on a 60-bp insertion in Solyc01g006550.2.1, making the products in CGN18423 and Moneymaker different in size. This marker was tested in $F_{2}$ plants and different $F_{3}$ lines. Only three $\mathrm{F}_{2}$ plants showed an unexpected genotype. These three $F_{2}$ plants were susceptible in the inoculation test, but showed the $\mathrm{F}_{1}$ genotype in the P7 test. Hammond-Kosack and Jones [2] suggested that the increased C. fulvum invasion of host tissue and the higher titer of intercellular fluid required to elicit a necrotic or chlorotic response in lines where a $C f$ gene was present in a heterozygous state indicates that $C f$ genes are incompletely dominant. The $C f-19$ gene may also be slightly affected by incompletely dominant inheritance, leading to a higher disease severity score for some heterozygous plants, and these plants were divided into the susceptible bulk. Although not all samples gave consistent results in the inoculation and P7 tests, the veracity of P7 in genotype identification is sufficient for MAS breeding work.

\section{The combination of SLAF-seq and parental resequencing is effective for gene mapping}

The SLAF-seq method provides significant advantages for developing large numbers of trait-related markers and target gene mapping. Genome resequencing can provide more details about genome sequences than SLAF-seq, and this information is useful to improve the precision of gene mapping. In this study, three association regions totaling $13.57 \mathrm{Mb}$ in size were obtained in $\mathrm{F}_{2}$ bulk SLAF-seq analysis, and a 2.14-Mb region with seven candidate genes was finally identified based on further SNP analysis using data from parental resequencing. The results of qRT-PCR analysis showed the accuracy of the mapping region at the transcriptional level. The current study also indicates that the combination of $\mathrm{F}_{2}$ bulk SLAF-seq and parental resequencing is a good choice for both relatively low cost and high efficiency gene mapping and trait-related gene screening.

\section{Impact of the current work on plant breeding and resistance mechanism research}

Breeding to obtain resistant cultivars is an efficient method for the control of leaf mold outbreaks. The differentiation of $C$. fulvum physiological races is very rapid, and new $C f$ resistance genes are of great significance to breeding. $C f-19$ was located on the short arm of chromosome 1 and one candidate gene Solyc01g006550.2.1 was assigned to the $C f-4 / 9$ locus, while another candidate gene Solyc01g005870.1.1 was mapped close to the $C f-4 / 9$ locus, which indicates that the $C f-19$ gene may have a direct evolutionary relationship with $C f-4$ and $C f-9$. The characteristics of $C f-19$ gene introgression to other cultivars may be similar to those of $C f-4$ and $C f-9$ and we have lots of experience in breeding cultivars carrying the $C f-4$ or $C f-9$ gene. Our location results will help guide the breeding of cultivars carrying the $C f-19$ gene. The $C f$ gene family is important for studying plant resistance $(R)$ gene evolution and R/Avr interaction mechanisms [26-28]. The candidate gene Solyc01g006550.2.1 is a new member of the $H c r 9 s$ and has a close evolutionary relationship with $C f-4$ and $C f$-9. It provides us a new gene material for plant resistance mechanism and $R$ gene evolution research. The results we obtained in the present study were based on genetic mapping, expression pattern analysis and sequence analysis, but to clone the $C f-19$ gene correctly, we still need functional verification such as virus-induced gene silencing (VIGS) and transgenic experiments.

\section{Conclusions}

In this study, we used $\mathrm{F}_{2}$ SLAF-seq and parental resequencing to locate the $C f-19$ gene. A total of 4108 Diff-markers were obtained. Three association regions consisting of 203 Diff-markers and 244 genes were found on chromosome 1. A 2.14-Mb region with seven candidate genes on the short arm was obtained through SNP analysis, and the candidate genes Solyc01g0 06550.2.1 and Solyc01g005870.1.1 were identified from these seven genes by qRT-PCR analysis. The candidate gene Solyc01g006550.2.1 is a new member of the Hcr9s in the $C f-4 / 9$ locus. A SCAR marker (P7) that was cosegregant with the resistance trait was developed that can be used in MAS breeding work. These results provide a basis for $C f-19$ gene cloning and application of the $C f-19$ gene in breeding.

\section{Methods}

\section{Plant materials and nucleotide extraction}

The resistant line S. lycopersicum CGN18423 containing the $C f-19$ gene (kindly provided by the Institute of Vegetable and Flowers, Chinese Academy of Agricultural Sciences) was crossed with the susceptible line $S$. lycopersicum Moneymaker. The resulting $\mathrm{F}_{1}$ plants were self-crossed and backcrossed with Moneymaker to obtain $\mathrm{F}_{2}$ and $\mathrm{BC}_{1}$. Three $\mathrm{F}_{2}$ plants with different genotypes were self-crossed to obtain $\mathrm{F}_{3}$ lines. All plants were grown at the Horticultural Experimental Station of Northeast Agricultural University.

At the five- to six-leaf stage, the seedlings of CGN18423, Moneymaker, $\mathrm{F}_{1}, \mathrm{~F}_{2}, \mathrm{BC}_{1}$ and $\mathrm{F}_{3}$ plants were inoculated with $C$. fulvum race 1.2.3.4. The plants were assessed for disease severity at 15 DAI. Inoculation and assessment of disease severity ratings were performed as described by Wang et al. [6]. The disease severity symptoms of the plants were converted to a disease score of 
0-9 points-0 points: no visible signs of infection; 1 point: 1-mm-diameter white spots or necrotic spots on the upper sides of leaves; 3 points: 2 to 3-mm-diameter yellow spots on the upper sides of leaves, some white mold on the lower sides of leaves, no spores formed; 5 points: 5 to 8 -mm-diameter yellow spots on the upper sides of leaves, abundant white mold on the lower sides of leaves, a few spores formed; 7 points: 5 to $8-\mathrm{mm}$ diameter yellow spots on the upper sides of leaves, some black mold, many spores on the lower sides of leaves, also some black mold and no spores on the upper sides of leaves; 9 points: masses of spores formed on both sides of the leaves. Plants with a disease score of 0 to 3 points were classified as resistant whereas those with a score of 5 to 9 points were classified as susceptible.

Based on the inoculation results, 50 resistant and 50 susceptible plants were selected from the $F_{2}$ generation as two bulks for bulked segregate analysis (BSA) [29]. The bulked DNA samples were prepared by mixing an equal ratio of DNA extracted from $200 \mathrm{mg}$ leaf samples using the cetyl trimethyl ammonium bromide (CTAB) method [30] with some modification [6]. These DNA samples were used for SLAF-seq analysis. DNA from the parents (CGN18423 and Moneymaker) and $F_{3}$ lines was also prepared using the $\mathrm{CTAB}$ method for parental resequencing and marker testing, respectively.

Leaf samples of CGN18423 and Moneymaker were collected at $0,4,5,7,10,13,17$ and 21 DAI for qRTPCR analysis. Total RNA was extracted from leaf samples using a plant RNA mini kit (Watson, Beijing, China) according to the manufacturer's handbook. First-strand cDNA was synthesized using an M-MLVRTase cDNA synthesis kit (Takara, Dalian, China) according to the manufacturer's instructions.

\section{SLAF-seq and association analysis}

A pre-design SLAF experiment was performed as described by [31]. According to the pre-design scheme, purified DNA was digested into DNA fragments of 364$464 \mathrm{bp}$ in size, using an appropriate restriction enzyme (RsaI). All subsequent SLAF-seq procedures were carried out referring to Sun et al. [19]. In the association analysis, P stands for the susceptible parent Moneymaker, $M$ means the resistant parent CGN18423, aa represents the resistant pool and ab represents the susceptible pool. There are two genotypes for a single marker; aa1 and ab1 mean the depth of aa and ab samples with the first genotype, respectively, and aa2 and ab2 stand for the depth of aa and ab samples with the other genotype, respectively. These variables were used in the following calculations: aa index =aa1aa1/aa2; $\mathrm{ab}$ index $=\mathrm{ab} 1 \mathrm{ab} 1 / \mathrm{ab} 2 ; \Delta \mathrm{SNP}$ index $=\mathrm{aa}$ index $-\mathrm{ab}$ index. The resistance phenotype is dominant, and the ratios of the two genotypes that can be detected are the same in unrelated markers, so the $\triangle \mathrm{SNP}$ index of unrelated markers is equal to 0 . Only one genotype of the $a b$ sample can be detected in related markers; we stipulated that ab index equaled 0 or 1 and aa index had a value of 0.66 or 0.33 , so the $\triangle \mathrm{SNP}$ index of related markers was equal to -0.66 or 0.66 , respectively. We calculated the $\triangle \mathrm{SNP}$ index value of all markers and fitted these data; $95 \%$ of the markers had fitting values greater than 0.3868831 , so we chose this value to screen regions related to our target trait. Markers with fitting values higher than 0.3868831 were marked as Diff_Markers.

Table 4 Primers used for qRT-PCR analysis and candidate loci sequencing

\begin{tabular}{|c|c|c|}
\hline Primer name & Forward primer sequence $\left(5^{\prime}-3^{\prime}\right)$ & Reverse primer sequence $\left(5^{\prime}-3^{\prime}\right)$ \\
\hline \multicolumn{3}{|c|}{ qRT-PCR primers } \\
\hline qRT-5730A & CTGGATCGCCCATTTCACCT & CCCATGTAACTCTGTGCCTGA \\
\hline qRT-5760A & TCTCATGGGTTACGGTTGTGG & TTCAAGTTTGTGTCTGATTTCTGT \\
\hline qRT-5780A & СTCACCTCATTTGTGCCCCA & ATCACTTGCCCTGTCGTCTC \\
\hline qRT-5870A & ATTGGCGGTGAAATCCCACA & AACCATAACCCACGAGCACC \\
\hline qRT-6550A & TाTAGTGCAGAAATGGGTTGTGT & CACTTGTCCTGTCGTCTCGT \\
\hline qRT-8390A & TCAGATGCTATTAGATTCAGACCTC & GTGGGAGACCTAACAGTGAGC \\
\hline qRT-8410A & AGTTCTCAGTATCCAGAGTAGCCT & AGAAATTGGTCCTTCGAGATGGT \\
\hline EFa1 & CCACCAATCTTGTACACATCC & AGACCACCAAGTACTACTGCAC \\
\hline \multicolumn{3}{|c|}{ Sequencing primers } \\
\hline$C f-4 / 9-1$ & TTCGTAGACCAAATTAGCT & CTAGTTGACCAATGTGAAG \\
\hline Cf-4/9-2 & TGGTCATTGATCTTCTAA & ACAGTTGGATTAGTAGGATTAC \\
\hline$C f-4 / 9-3$ & AAGGTGTACCTTATAGCAT & GCGTTCGAGTGTTTCGGGGAA \\
\hline S5870A & AACAAAAGTCTTAATTATTTATA & ACCAATTTCCCAGTTCTCGATA \\
\hline S5870B & GCTTATTCCAGTCCAATT & CACTTAATGCATTGGCAAAAAGA \\
\hline
\end{tabular}




\section{Parental genome sequencing and data analysis}

DNA from CGN18423 and Moneymaker was used in this part of analysis. All steps including sequencing, reads mapping, and analysis of SNP and insertion/deletion (InDel) polymorphisms were carried out according to Bai et al. [32]. The tomato genome sequence of Heinz 1706 [33] was used as the reference genome for reads mapping. SNPs in the association regions obtained from parental resequencing were analyzed in combination with those from $\mathrm{F}_{2}$ bulk SLAF-seq. SNPs that showed the Moneymaker base type in the susceptible pool, and both parental base types in the resistant pool were screened out to further narrow the mapping regions.

\section{Gene annotation and candidate gene screening}

Genes in the final mapping regions were annotated according to the tomato genome annotations in the National Center for Biotechnology Information (NCBI) (http://www.ncbi.nlm.nih.gov/BLAST/) and SOL Genomics Network (SGN) (http://solgenomics.net/) websites. All genes with $C f$-type characters in function (receptorlike) and structure (LRR domain) were selected as candidate genes.

\section{Quantitative real-time PCR analysis of candidate genes} qRT-PCR was carried out for all seven candidate genes. The primers (Table 4) for qRT-PCR were designed using the Primer 5.0 software. The qRT-PCR reaction was performed using an iQ5 system (Bio-Rad, USA). The reaction mixture contained $10 \mu \mathrm{L}$ of $2 \times$ TransStart Top Green qPCR SuperMix (TransGen, China), $10 \mathrm{pM}$ of each primer, $2 \mu \mathrm{L}$ of cDNA templates (1:10 dilution), and sterile distilled water to make up a total volume of $20 \mu \mathrm{L}$. The thermal conditions (two-step method) were as of $95{ }^{\circ} \mathrm{C}$ for $10 \mathrm{~s}, \mathrm{~T}_{\mathrm{m}}$ temperature for $30 \mathrm{~s}$. To detect primer dimerization or other artifacts of amplification, a melting-curve analysis was performed immediately after completion of the RT-PCR $\left(95^{\circ} \mathrm{C}\right.$ for $15 \mathrm{~s}$ and $55^{\circ} \mathrm{C}$ for $15 \mathrm{~s}$, followed by a slow increase of temperature by $0.5{ }^{\circ} \mathrm{C}$ per cycle to $95{ }^{\circ} \mathrm{C}$ with continuous measurement of fluorescence). The data were analyzed using the $2^{-\Delta \Delta C T}$ method [34] with EF 1 as a reference gene for normalization [35].

\section{Candidate loci sequencing and DNA sequence analysis}

To obtain detailed DNA sequence information, primers (Table 4) were designed for the candidate gene loci Solyc01g005870.1.1 and Solyc01g006550.2.1 using the Primer 5.0 software. The template sequences were from the reference genome sequence of SGN. The PCR products of CGN18423 and Moneymaker were purified with a PCR purification kit (Takara). The purified products were cloned into the pMD18-T vector (Takara) and sequenced. The DNA sequences we obtained were submitted to the NCBI database and analyzed using the Blast (http:// blast.ncbi.nlm.nih.gov/Blast.cgi) and Open Reading Frame Finder (ORF Finder) (http://www.ncbi.nlm.nih.gov/gorf/ gorf.html) tools of NCBI. Gene structure analysis was performed using the online tool SMART (http://smart.embl-heidelberg.de/). Other known $C f$ genes in the Cf-4/9 locus (GenBank: AY639604.1, AJ002235.1, AJ002236.1) were also analyzed in combination with the candidate genes in this study to reveal evolutionary relationships.

\section{Marker developing and linkage analysis}

Six markers including cleaved amplified polymorphic sequence (CAPS) markers and SCAR markers were designed based on sequence mutations in or near the candidate gene loci. Marker primers were designed using the Primer 5.0 software. The markers were first screened using CGN18423, Moneymaker and the $F_{1}$ plants. Linkage analysis was then performed for these markers in an $\mathrm{F}_{2}$ population consisting of 345 plants. In this part, a special SCAR marker P7 was screened out. Therefore, three $F_{3}$ lines $\left(F_{3}-1\right.$, derived from an $F_{2}$ plant with the resistant homozygous genotype; $F_{3}-2$, derived from an $F_{2}$ plant with the resistant heterozygous genotype; $\mathrm{F}_{3}-3$, derived from an $\mathrm{F}_{2}$ plant with the susceptible homologous genotype; based on the P7 test), were used to further test the genotyping veracity of the marker P7.

\section{Availability of supporting data}

The data sets supporting the results of this article are included within the article and its additional files.

\section{Additional file}

Additional file 1: Table S1. Results from combined data analysis of parental resequencing and $F_{2}$ SLAF-seq. Forty-three SNPs that showed the Moneymaker base type in the susceptible pool, and both parental base types in the resistant pool were screened out. Of the 43 SNPS, 34 were distributed in an approximately 2.14-Mb region in association region I, one was in association region II and eight were in association region III. Seven Cf-type genes were identified in association region I. (DOCX $16 \mathrm{~kb}$ )

\begin{abstract}
Abbreviations
Avr: Avirulence gene; BSA: bulked segregate analysis; C. fulvum: Cladosporium fulvum; CAPS: cleaved amplified polymorphic sequences; CTAB: Cetyl

Trimethyl Ammonium Bromide; DAl: days after inoculation; Hcr2: homologues of Cladosporium resistance gene Cf-2; Hcr9: homologues of Cladosporium resistance gene (f-9; HR: hypersensitive response; InDel: insertion/deletion; LRR: leucine-rich repeat; MAS: marker assistant selection; NCBI: National Center for Biotechnology Information; ORF: open reading frame finder; qRTPCR: quantitative real-time PCR; QTL: quantitative trait loci; $R$ gene: resistance gene; SCAR: sequence characterized amplified region; SGN: SOL Genomics Network; SLAF-seq: specific-locus amplified fragment sequencing; SNP: single nucleotide polymorphism; VIGS: virus induced gene silencing.
\end{abstract}

Competing interests

The authors declare that they have no competing interests. 


\section{Authors' contributions}

TZ conceived this study, performed the experiments of artificial inoculation DNA and RNA extraction, high-throughput sequencing data analysis, expression pattern analysis, candidate gene sequencing and candidate gene sequence analysis, and wrote the manuscript. XX organized the entire project and helped to prepare the manuscript. JJ, GL and SH prepared the plant material. HZ, XC and $J L$ prepared chemical reagent and other materials for this project. All authors read and approved the final manuscript.

\section{Acknowledgements}

This research was supported by the National Natural Science Foundation of China (NSFC; 31272171), China Agriculture Research System (CARS-25), and Heilongj Science Foundation for Distinguished Young Scholars (JC201204).

Received: 29 October 2015 Accepted: 17 February 2016 Published online: 25 February 2016

\section{References}

1. Thomma BP, Van Esse HP, Crous PW, De Wit PJ. Cladosporium fulvum (syn. Passalora fulva), a highly specialized plant pathogen as a model for functional studies on plant pathogenic Mycosphaerellaceae. Mol Plant Pathol. 2005:6(4):379-93.

2. Hammond-Kosack KE, Jones JD. Incomplete dominance of tomato Cf genes for resistance to Cladosporium fulvum. Mol Plant Microbe In. 1994:7:58-70.

3. Joosten $\mathrm{MH}$, De Wit PJ. The tomato-Cladosporium fulvum interaction: a versatile experimental system to study plant-pathogen interactions. Annu Rev Phytopathol. 1999;37:335-67.

4. Rivas S, Thomas CM. Molecular interactions between tomato and the leaf mold pathogen Cladosporium fulvum. Annu Rev Phytopathol. 2005;43:395-436.

5. Lindhout P, Korta W, Cislik M, Vos I, Gerlagh T. Further identification of races of Cladosporium fulvum (Fulvia fulva) on tomato originating from the Netherlands, France and Poland. Neth J Plant Path. 1989;95:143-8.

6. Wang A, Meng F, Xu X, Wang Y, Li J. Development of molecular markers linked to Cladosporium fulvum resistant gene Cf-6 in tomato by RAPD and SSR methods. HortSci. 2007:42:11-5.

7. Parniske M, Hammond-Kosack KE, Golstein C, Thomas CM, Jones DA, Harrison $\mathrm{K}$, Jones JD. Novel disease resistance specificities result from sequence exchange between tandemly repeated genes at the Cf-4/9 locus of tomato. Cell. 1997:91:821-32.

8. Nekrasov V, Ludwig AA, Jones JD. CITRX thioredoxin is a putative adaptor protein connecting $C f-9$ and the ACIK1 protein kinase during the Cf-9/Avr9induced defence response. FEBS let. 2006:580:4236-41.

9. Gabriëls SH, Takken FL, Vossen JH, De Jong CF, Liu Q, Turk SC, Joosten MH. CDNA-AFLP combined with functional analysis reveals novel genes involved in the hypersensitive response. Mol Plant Microbe In. 2006;19:567-76.

10. Kanwar JS, Kerr EA, Harney PM. Linkage of Cf-1 to Cf-11 genes for resistance to leaf mold Cladosporium fulvum Cke. Rep Tomato Genet Coop. 1980;30:20-1.

11. Kanwar JS, Kerr EA, Harney PM. Linkage of the Cf-12 to Cf-24 genes for resistance to tomato leaf mold Cladosporium fulvum Cke. Rep Tomato Genet Coop. 1980;30:22-3.

12. Westerink N, Brandwagt BF, De Wit PJ, Joosten MH. Cladosporium fulvum circumvents the second functional resistance gene homologue at the Cf-4 locus (Hcr9-4E) by secretion of a stable avr4E isoform. Mol Microbiol. 2004;54:533-45.

13. Balint-Kurti PJ, Dixon MS, Jones DA, Norcott KA, Jones JDG. RFLP linkage analysis of the Cf-4 and Cf-9 genes for resistance to Cladosporium fulvum in tomato. Theor Appl Genet. 1994;88:691-700.

14. Dixon MS, Jones DA, Keddie JS, Thomas CM, Harrison K, Jones JDG. The tomato $\mathrm{Cf}$-2 disease resistance locus comprises two functional genes encoding leucine-rich repeat proteins. Cell. 1996;84:451-9.

15. Dixon MS, Hatzixanthis K, Jones DA, Harrison K, Jones JDG. The tomato Cf-5 disease resistance gene and six homologs show pronounced allelic variation in leucine rich repeat copy number. Plant Cell. 1998:10:1915-25.

16. Jones DA, Dickinson MJ, Balint-Kurti PJ, Dixon MS, Jones JDG. Two complex resistance loci revealed in tomato by classical and RFLP mapping of the Cf-2, Cf-4, Cf-5 and Cf-9 genes for resistance to Cladosporium fulvum. Mol Plant Microbe In. 1993;6:348-57.

17. Thomas CM, Jones DA, Parniske M, Harrison K Balint-Kurti PJ, Hatzixanthis K Jones JDG. Characterization of the tomato Cf-4 gene for resistance to Cladosporium fulvum identifies sequences that determine recognitional specificity in Cf-4 and Cf-9. Plant Cell. 1997;9:2209-24.
18. Zhao TT, Liu G, Li S, Li JF, Jiang JB, Zhang H, Kang LG, Chen XL, Xu XY. Differentially expressed gene transcripts related to the Cf-19-mediated resistance response to Cladosporium fulvum infection in tomato. Physiol Mol Plant P. 2015:89:8-15.

19. Sun X, Liu D, Zhang X, Li W, Liu H, Hong W, Zheng H. SLAF-seq: an efficient method of large-scale de novo SNP discovery and genotyping using high-throughput sequencing. PLoS One. 2013;8, e58700.

20. Zhang $Y$, Wang L, Xin H, Li D, Ma C, Ding X, Hong W, Zhang X. Construction of a high-density genetic map for sesame based on large scale marker development by specific length amplified fragment (SLAF) sequencing. BMC Plant Biol. 2013;13:141.

21. Kerr EA, Bailey DL. Resistance to Cladosporium fulvum Cke obtained from wild species of tomato. Can J Bot. 1964;42:1541-53.

22. Soumpourou E, lakovidis M, Chartrain L, Lyall V, Thomas CM. The Solanum pimpinellifolium Cf-ECP1 and Cf-ECP4 genes for resistance to Cladosporium fulvum are located at the Milky Way locus on the short arm of chromosome 1. Theor Appl Genet. 2007;115:1127-36.

23. Haanstra JPW, Laugé R, Meijer-Dekens F, Bonnema G, De Wit PJGM, Lindhout $P$. The Cf-ECP2 gene is linked to, but not part of, the Cf-4/Cf-9 cluster on the short arm of chromosome 1 in tomato. Mol Gen Genet. 1999: 262:839-45.

24. Yuan Y, Haanstra J, Lindhout $P$, Bonnema G. The Cladosporium fulvum resistance gene Cf-ECP3 is part of the Orion cluster on the short arm of tomato Chromosome 1. Mol Breed. 2002;10:45-50.

25. Haanstra JPW, Meijer-Dekens F, Lauge R, Seetanah DC, Joosten MHAJ, De Wit PJGM, Lindhout P. Mapping strategy for resistance genes against Cladosporium fulvum on the short arm of chromosome 1 of tomato: Cf-ECP5 near the Hcr9 Milky Way cluster. Theor Appl Genet. 2000;101:661-8.

26. Thomas CM, Dixon MS, Parniske M, Golstein C, Jones JDG. Genetic and molecular analysis of tomato Cf genes for resistance to Cladosporium fulvum. Philosophical Transactions of the Royal Society of London. Ser B Biol Sci. 1998;353:1413-24.

27. Wulff BBH, Chakrabarti A, Jones DA. Recognitional specificity and evolution in the tomato-Cladosporium fulvum pathosystem. Mol Plant Microbe In. 2009:22:1191-202.

28. Cai $X$, Takken FL, Joosten MH, De Wit PJ. Specific recognition of Avr4 and Avr9 results in distinct patterns of hypersensitive cell death in tomato, but similar patterns of defence-related gene expression. Mol Plant Pathol. 2001;2(2):77e86.

29. Michelmore R, Paran I, Kesseli RV. Identification of markers linked to diseaseresistance genes by bulked segregant analysis: a rapid method to detect markers in specific genomic regions by using segregating populations. Proc Natl Acad Sci U S A. 1991;88:9828-32.

30. Fulton TM, Chunwongse J, Tanksley SD. Microprep protocol for extraction of DNA from tomato and other herbaceous plants. Plant Mol Biol Rpt. 1995:13:207-9.

31. Chao X, Chen L, Rong TZ, Li R, Xiang Y, Wang P, Liu CH, Dong XQ, Liu B, Zhao D, Wei RJ, Lan $H$. Identification of a new maize inflorescence meristem mutant and association analysis using SLAF-seq method. Euphytica. 2015;202(1):1-10

32. Bai H, Cao Y, Quan J, Dong L, Li Z, Zhu Y, Li D. Identifying the genomewide sequence variations and developing new molecular markers for genetics research by re-sequencing a landrace cultivar of foxtail millet. PLoS One. 2013;8, e73514.

33. Consortium, The Tomato Genome. The tomato genome sequence provides insights into fleshy fruit evolution. Nature. 2012;485(7400):635-41.

34. Livak KJ, Schmittgen TD. Analysis of relative gene expression data using real-time quantitative PCR and the $2^{-\Delta \Delta C T}$ method. Methods. 2001;25:402-8.

35. Rotenberg D, Thompson TS, German TL, Willis DK. Methods for effective real-time RT-PCR analysis of virus-induced gene silencing. J Virol Methods. 2006:138:49-59. 